\title{
Relationship between Glycemic Control in Type II Diabetes Mellitus and Anaemia
}

\author{
Anand Dev ${ }^{1}$, Nitali Arun ${ }^{2}$ \\ ${ }^{1}$ Assistant Professor, Department of Internal Medicine, TMMC \& RC, Moradabad, ${ }^{2}$ Assistant Professor, Department of Microbiology, TMMC \&RC.
}

\section{Abstract}

Background: Chronic hyperglycemia in uncontrolled diabetes is related to higher incidence of anaemia which goes unrecognized prior to the development of chronic renal failure.This study was conducted to know the prevalence of Anaemia in persons with with Type II Diabetes Mellitus,in relation to glycemic control using HbA1c as a tool to it. Subjects and Methods: 60 Diabetic subjects were divided into two groups of 30 each based on their glycemic control(group A,with poor glycemic control and group B with good glycemic control taking 7\% Hba1c as cut off value),incidence of anaemia was measured and compared among them and also with 30 age and sex matched healthy non Diabetic controls. Results: Incidence of anaemia was found to be significantly higher in diabetics group as compared to non diabetics and among diabetics it was significantly higher in uncontrolled group as compared to group with controlled diabetes. Conclusion: Good glycemic control in diabetes is associated with a better haemoglobin levels, hence it is desirable to evaluate the haemoglobin level often to monitor the micro and macrovascular complications of diabetes even when the renal parameters are normal.

Keywords: Diabetes, Anaemia, Glycemic control, HbA1c, micro and macro vascular complications.

Corresponding Author: Dr. Nitali Arun, Assistant Professor, Department of Microbiology, Teerthanker Mahaveer Medical College \& Research Centre, Moradabad.

Received: November 2018

Accepted: December 2018

\section{Introduction}

Diabetes mellitus is a group of metabolic diseases characterized by elevated blood glucose levels (hyperglycemia) resulting from defects in insulin secretion, insulin action or both. Chronic hyperglycemia is associated with microvascular and macrovascular complications that include neuropathy, retinopathy, coronary artery disease, and stroke. ${ }^{[1]}$

The prevalence of Type II Diabetes Mellitus worldwide is on a rise and has reached epidemic proportions in many countries. The total number of adult patients suffering from Type II Diabetes Mellitus has reached 366 million in 2011, ${ }^{[2]}$ Globally an estimated 415 million adults were living with diabetes in 2015 , and it is on surge to reach to 552 million by $2030 .^{[3,4]}$ China tops the world among diabetes affected countries having a total of around 90 million. Type II Diabetes Mellitus patients. India follows China with prevalence of 69 million. ${ }^{[5]}$ The number of Type II Diabetes Mellitus cases in India is expected to rise to 101.2 million by 2030. ${ }^{[3]}$

Glycemic control is considered as the main therapeutic goal for prevention of organ damage and other complications of diabetes and gives an accurate estimate of the average plasma glucose levels from past 8 to 12 weeks. Glycemic control can be considered as poor and good based on the values of HbA1c. Desirable values of HbA1c is to be maintained suggesting a good glycemic control is $7 \%$. Values of $\mathrm{HbA} 1 \mathrm{c}$ more than $7 \%$ is considered as poor glycemic control in Type II Diabetes Mellitus. ${ }^{[6,7]}$

There are an increasing number of patients with diabetes who have been found to be anaemic without any evidence of any chronic renal disease. In a study conducted by Adetunji, Olujohungbe and Ronand, it was evident that anaemia is commonly associated in patients with diabetes mellitus. They studied the association with diabetes and anaemia in over 500 patients and ultimately concluded that anaemia was very common among the study group. ${ }^{[8]}$

Anaemia may be more common in diabetes and develop earlier than in patients with renal impairment from other causes. However, patients with diabetes may be more vulnerable to the effects of anaemia because many also have significant cardiovascular disease and hypoxia - induced organ damage. ${ }^{[9]}$

Many factors have been suggested as the reason for the earlier onset of anaemia in patients with diabetes, including severe symptomatic autonomic neuropathy, causing efferent sympathetic denervation of the kidney and loss of appropriate erythropoietin production; damage to the renal interstitium; systemic inflammation; and inhibition of erythropoietin release. ${ }^{[10]}$

Following are the cheaf factors responsible for anaemia in diabetics as proposed and studied thoroughly:Diabetic Nephropathy,Renal Tubular Dysfunction,TubularIschaemia,Erythropoietin-deficiency,Chronic 
inflammation,Advance glycation end products,Oxidative stress,Abnormal hemetinic absorption,Medications,Diabetic neuropathy. ${ }^{[11]}$

The present study was aimed to find an association between anaemia and Type II Diabetes Mellitus.It also intend to find the relation of anaemia and degree of glycemic control and the morphological type of anemia most commonly associated.

\section{Subjects and Methods}

The present study was carried out in TMU Moradabad. The study was a longitudinal case control study over a period of one and half year, after obtaining ethical clearance from the Institutional Ethics Committee and obtaining written consents from the participants.60 diagnosed cases of Type II Diabetes Mellitus were divided into two groups of 30 each based on glycemic control depending on HbA1c level according to ADA criteria for control of Diabetes, Group "A" with poor glycemic control(HbA1c $>7 \%)$ and Group "B" with good glycemic control(HbA1c<7\%). They were compared among themselves and with another 30 healthy age and sex matched Non Diabetic control(Group "C").

\section{Inclusion Criteria:}

- Males and Females aged 20 years and above.

- Clinically proven cases of Type II Diabetes Mellitus with Anaemia

(Diabetes Diagnosed as per ADA criteria i.e. symptoms of diabetes with random blood sugar $>200 \mathrm{mg} / \mathrm{dl}$ or fasting blood sugar $>126 \mathrm{mg} / \mathrm{dl}$ or $\mathrm{HbA} 1 \mathrm{c}>6.5 \%$ or 2 hour plasma glucose $>200 \mathrm{mg} / \mathrm{dl}$ during an oral glucose tolerance test. Anaemia is diagnosed as per WHO criteria i.e. $\mathrm{Hb}<13 \mathrm{gm} / \mathrm{dl}$ for male and $\mathrm{Hb}<12 \mathrm{gm} / \mathrm{dl}$ for female).

\section{Exclusion criteria:}

- Other causes of Anaemia not associated with Diabetes

- Associated co- morbid conditions like Hypertension, Congestive Cardiac Failure, Chronic Renal Failure, Stroke, Haemorrhological Disorders and Malignancy.

- Obstetric and Gynaecological Disorders

- Patients on Hormone Therapy

- Pregnancy

- Patient not willing to give consent for the study.

Subjects were enrolled in the study based on the inclusion and exclusion criteria. The selected subjects were briefed about the nature of the study and a written informed consent was obtained before the subject was enrolled in this study.

Demographic data like gender, age were collected along with relevant history and recorded on predesigned proforma. A thorough clinical examination was conducted and findings were also recorded. Anthropometry including height $(\mathrm{cm})$, weight(Kgs) was measured and BMI was calculated based on quetelet index; $\mathrm{BMI}=$ weight $(\mathrm{Kg}) /$ height $(\mathrm{mts}) .{ }^{[2]}$

The patient were then instructed to come the next day in an 8 hour fasting state for the blood investigations.

Blood sample from the study and control group was drawn under complete aseptic precautions. Fasting and two hours post prandial blood sample were collected for analysis in vacuum evacuated tubes as follows:
1. Clot activator containing vacuum evacuated tubes for estimation of Complete Haemogram, Peripheral Blood Smear, Renal Function Test.

2. Fluorides EDTA vacuum evacuated tubes for estimation of blood glucose and glycosylated haemoglobin.

3. Sterile Urine container for the analysis of Urine Microscopy, urine culture and sensitivity.

4. Sterile stool container for the analysis of Stool Microscopy (for occult blood)

The data collected were analyzed and expressed as Mean \pm SD. One way Analysis of variance (one way ANOVA), Pearson's correlation test were used in the present study. Alongwith logical regression to obtain relative risk and odd's ratio. Statistical software SPSS 20.0 was used for the analysis of the data and Microsoft Word and Excel to generate graphs and tables. Level of Significance: P $<0.05$ was considered as significant while analyzing the data.

\section{Results}

60 Diabetic SUBJECTS were divided into two groups based on their glycemic control; Poor glycemic control diabetic group (HbA1c > $7 \%$ ) - Group A, Better glycemic control diabetic group ( $\mathrm{HbA} 1 \mathrm{c}<7 \%$ ) - Group B and normal healthy CONTROLS were taken - Group C (another 30 patients). The results were tabulated and analyzed.

In the present study, there were 37 female and 53 male subjects, mean ages of all the groups are similar and comparable and there was no significant difference, as shown in following table:

Table 1: Age Distribution.
\begin{tabular}{|c|c|c|c|}
\hline & Group A & Group B & Group C \\
\hline Age (Years) & $58.3 \pm 7.93$ & $55.13 \pm 12.49$ & $51.90 \pm 10.46$ \\
\hline
\end{tabular}

Height, Weight and Body Mass Index were measured and documented in the present study,there was no significant difference between them. The results are shown in the table below:

Table 2: Demographic data.
\begin{tabular}{|c|c|c|c|}
\hline & Group A & Group B & Group C \\
\hline Height $(\mathrm{cms})$ & $161.9 \pm 6.79$ & $165.2 \pm 5.83$ & $164 \pm 7.55$ \\
\hline Weight $(\mathrm{Kg})$ & $61.46 \pm 8.84$ & $67.03 \pm 9.74$ & $64.23 \pm 6.81$ \\
\hline BMI $(\mathrm{Kg} / \mathrm{m} 2)$ & $24.53 \pm 2.02$ & $24.46 \pm 2.75$ & $23.90 \pm 2.24$ \\
\hline
\end{tabular}

- Anaemia was detected in the $40 \%$ of diabetic patients and majority was in the age group 50-60 years, it was found in $25 \%$ of the group with uncontrolled diabetes as compared to $15 \%$ in the controlled diabetic group. Haemoglobin was significantly lower i.e $11.75 \pm 1.78$ in group with uncontrolled diabetes as compared to group of controlled diabetes i.e12.67 \pm 1.75 which was further significantly lower than in control non diabetic group i.e13.44 \pm 1.38. Hence In the diabetic group, better glycemic control group had a better haemoglobin percentage as compared to poor glycemic control which was statistically significant $(\mathrm{P}<$ 0.05).The above findings are tabulated and graphically represented below:

Table 3: Glycemic Status and Anaemia.

\section{Anaemia}

Present

\section{Anaemia}

Absent

Total 


\begin{tabular}{|c|c|c|c|}
\hline HbA1c $>7$ & 15 & 15 & 30 \\
\hline HbA1c $<7$ & 9 & 21 & 30 \\
\hline TOTAL & 24 & 36 & 60 \\
\hline
\end{tabular}

From the above $2 \times 2$ Contingency Table after calculating relative risk, it comes out to be 1.67 and odds ratio to be 2.33 , which suggests that anaemia is at least 1.6 times more common in poorly control diabetics than well controlled.

Among the diabetics with anaemia there were 12 male and 12 females, hence there was no difference in incidence between diabetic male and females.

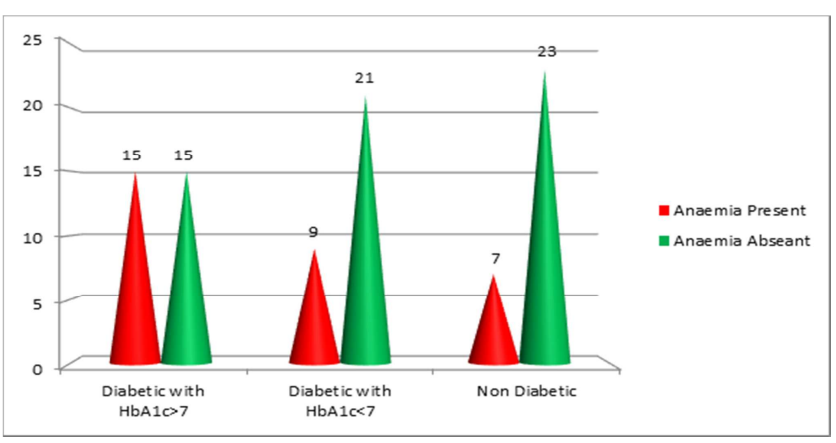

In the below mentioned table, values in Mean \pm SD. * indicates comparison with Group B with $\mathrm{p}$ value $<0.05$. \# indicates comparison with Group $\mathrm{C}$ with $\mathrm{P}$ value $<0.001$. \$ indicates comparison with Group A with p value $<0.001$. @ indicates comparison with Group $\mathrm{C}$ with $\mathrm{p}$ value $<0.01$.All calculations done using unpaired t tests.

\section{Table 4: Comparison of Haemoglobin and HbA1C.}

\begin{tabular}{|c|c|c|c|}
\hline & Group A & Group B & Group C \\
\hline $\mathrm{HbA1c}(\%)$ & $8.4 \pm 1.21$ & $6.64 \pm 0.18 \$ @$ & $6.08 \pm 0.23 \$$ \\
\hline $\mathrm{Hb} \%$ & $11.75 \pm 1.78^{*} \#$ & $12.67 \pm 1.75$ & $13.44 \pm 1.38^{*}$ \\
\hline
\end{tabular}

Majority of the anaemia is normocytic i.e 16 out of 20 in diabetes group (66.6\%) as compaered to microcytic (29\%) and 1 megaloblastic $(\sim 4 \%)$.In control group majority of anaemia 5 out of $7(71 \%)$ is in female and majority is of normocytic $(57 \%)$ type.

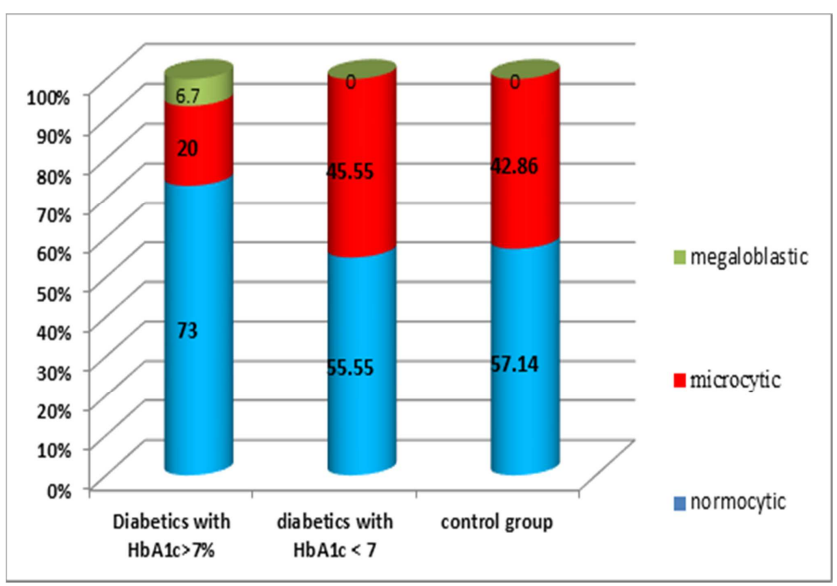

Fasting and post prandial blood sugar was significantly higher in the diabetic group compared with the normal controls. Among the diabetics FBS and PPBS was significantly higher in the poor glycemic control group than the good glycemic control group.
Table 5: Comparison of blood sugar.

\begin{tabular}{|c|c|c|c|}
\hline & Group A & Group B & Group C \\
\hline FBS (mg/dl) & $222.4 \pm 55.09$ & $131.5 \pm 25.48$ & $97.53 \pm 10.43$ \\
\hline PPBS (mg/dl) & $257.36 \pm 62.42$ & $173.3 \pm 31.42$ & $125.7 \pm 17.03$ \\
\hline
\end{tabular}

There is significant $(\mathrm{p}<0.001)$ difference between group A and group $\mathrm{C}$ and also between group $\mathrm{B}$ and group $\mathrm{C}(\mathrm{p}<0.01)$. There is a statistically significant negative correlation between Haemoglobin percentage and $\mathrm{HbA} 1 \mathrm{c}$, with Pearson's correlation co-efficient being -0.6880 .

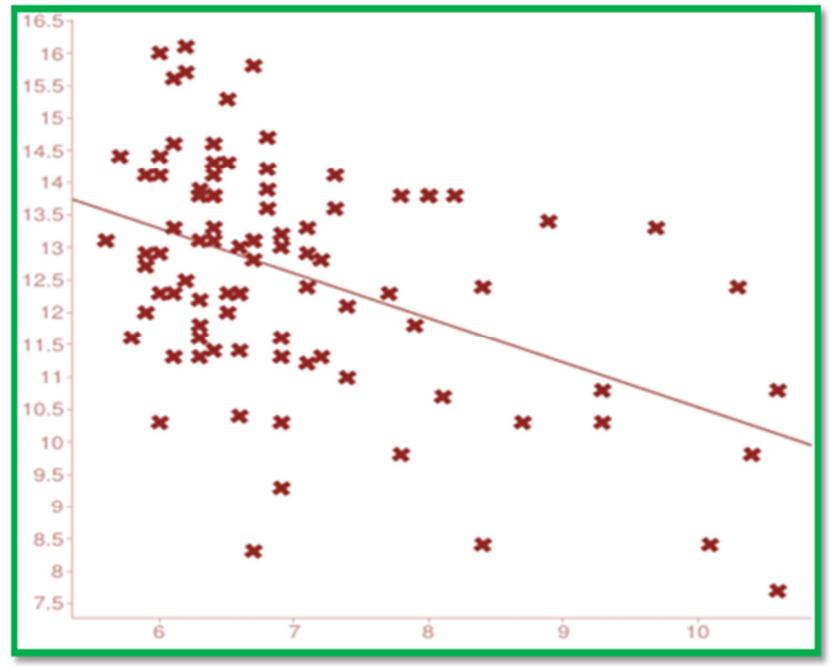

Renal parameters, standard routine examination and stool examination were within normal range for all the groups.

\section{Discussion}

In the present study there was significant prevalence of unrecognized anaemia was found among diabetics as compared to non diabetics, which was significantly higher among uncontrolled diabetes than with controlled hyperglycemia, even in the patients with normal renal function Al-khoury et al demonstrated that for each CKD stage, haemoglobin is $1 \mathrm{~g} / \mathrm{dl}$ lower in patients with diabetes than in non-diabetic population. There was statistically significant negative correlation between blood sugar level and haemoglobin as well as significant correlation between haemoglobin and HbAlc as shown by the present study. As compared to the study done by Merlin C Thomas et al, as Unrecognised anaemia in patients with Diabetes in 2003, the study showed weak association of haemoglobin with $\mathrm{HbA1c}$ ( 2\% $\mathrm{Hb}$ variance), when confounding factors like ACE inhibitor, any degree of retinopathy were adjusted for GFR showed weak correlation as opposed to the present study.9The present study does not show any difference between incidence of anaemia with diabetes in male as compared to female, a similar study by Noorul Ameen at el for the prevalence of anaemia in uncontrolled diabetes Type II incidence of diabetes was found to be higher (67\%) in females as compared to males.The majority of the anaemia showed normocytic normochromic blood picture as compared to microcytic hypochromic or megaloblastic. In a study done by Alap L Christy et al for influence of Iron Deficiency Anaemia on Haemoglobin A1c Levels in 
Diabetic individuals with controlled Plasma Glucose Levels, it was found that elevated HbAlc $(6.8 \pm 1.4 \%)$ in irondeficient individuals as compared to controls, that infer microcytic anaemia being more common.

\section{Conclusion}

To conclude anaemia is not a very uncommon finding in Type II Diabetes Mellitus patients when compared with the general population. Anaemia in patients with diabetes mellitus might contribute to pathogenesis and progression of cardiovascular disease and aggravate diabetic nephropathy and retinopathy. However, an emphasis on regular screening for anaemia, alongside that for other diabetes-related complications, might help to delay the progression of vascular complication in these patients Further good glycemic control in diabetes mellitus is associated with a better haemoglobin levels and a lesser incidence of anaemia. Hence in diabetic patients, it would be desirable to evaluate the haemoglobin levels often, even when the renal parameters are within the normal limits for a better quality of life.

\section{References}

1. Loghmani E. Diabetes Mellitis: Type 1 And Type 2. 2005 [cited 2014 Sep 15];

2. Ambady Ramachandran, Ananth Samith Shetty, Arun Nanditha, Chamukuttan, Snehalatha. Type II Diabetes in India: Challenges and possible solutions. In: Muruganathan A, editor. Medicine Update. Volume 23; New delhi: M/s Jaypee Brothers Medical Publishers (P) Ltd; 2013

3. Wild S, Sicree R, Roglic G, King H, Green A. Global prevalence of diabetes estimates for the year 2000 and projections for 2030. Diabetes Care 2004 May; 27(5):1047-53

4. WHO annual data from periodicals 2016.pg21-25

5. International diabetic federation report 2015

6. American Diabetes Education. Diagnosis and classification of diabetes mellitus. Diabetes care 37; 2014 Jan; suppl:s5-10

7. Powers AC. Diabetes Mellitus. In: Fauci AS, Braunwald E, Kasper DL, Hauser SL, Longo DL, Jameson JL et al. editors, Harrison's principles of Internal Medicine. 19th ed. USA: Mc Graw Hill: 2015: p: $2403-2405$

8. Adetunji, FWACP, A Olujohungbe, MRCP J.Ronand Prevalence and characteristic of anaemia in Diabetes Practical Diabetes, International Journal, Apr: 2008; 25. 1.

9. Merlin C Thomas, Richard J. MacIsaac, Con Tsalamandris, David Power, George Jerums. Unrecognized anaemia in patients with Diabetes. Diabetes Care. April 2003; 4: 1164 - 1169

10. Katherine J. Craig, John D. Williams, Stephen G.Riley, Hilary Smith, David R.Owens, Debbe Worthing et al. anaemia and Diabetes in the Absence of Nephropathy. Diabetes Care. May 2005; 28(5): 1118-1123

11. Dhruv K Singh,Peter Winocour and Ken Farrington; Erythropoietin stress and anaemia in Diabetes Mellitus: Natures Review of Endocrinology.2009; 5,204-210

Copyright: () the author(s), publisher. Academia Journal of Medicine is an Official Publication of "Society for Health Care \& Research Development". It is an open-access article distributed under the terms of the Creative Commons Attribution Non-Commercial License, which permits unrestricted non-commercial use, distribution, and reproduction in any medium, provided the original work is properly cited.

How to cite this article: Dev A, Arun N. Relationship between Glycemic Control in Type II Diabetes Mellitus and Anaemia. Acad. J Med. 2018;1(2):12-15.

DOI: dx.doi.org/10.21276/ajm.2018.1.2.4

Source of Support: Nil, Conflict of Interest: None declared. 